\title{
SAFE LABORATORY PRACTICES IN THE LIGHT OF COVID-19 PANDEMIC: WAY FORWARD IN A RESOURCE LIMITED SETTING
}

Odega K.I., Ehijie I., Ibadin, E.E., Idomeh F. A., Odega D.E

University Of Benin Teaching Hospital, Histopathology and Morbid Anatomy Department Benin City, Edo State, Nigeria.

University of Benin Teaching Hospital, Medical Laboratory Services, Chemical Pathology Unit, Benin City, Edo State, Nigeria

University of Benin Teaching Hospital, Medical Laboratory Services, Haematology and Blood Transfusion Unit, Benin City, Edo State, Nigeria

University of Benin Teaching Hospital, Medical Laboratory Services, Medical Microbiology Unit Benin City, Edo State, Nigeria

University of Benin Teaching Hospital, Human Reproductive Research Programme/In-vitro Fertilization Unit, Benin City, Edo State, Nigeria

\section{ABSTRACT}

The 2019 Coronavirus pandemic which was initially referred to as 2019-nCoV, was first identified in Wuhan, China. Early response from the Chinese government included quarantine of infected persons, isolation and total lockdown of Wuhan province to prevent further spread. With the spread of the disease across national borders and declaration of the disease as a global pandemic, there has been a robust response by the international community to contain this deadly virus and prevent its further spread worldwide. Africa is not left out of this rampaging pandemic with documented cases in over 40 countries and still rising. Although extensive studies have been carried out on the novel SARS-CoV-2 on its pathogenesis, mode of infection and virulence but much is still unknown. However, potentially infectious samples are received routinely in the medical laboratory for analysis. This technical note reviews good laboratory practice (GLP) and processes across the different specialities of Medical Laboratory practice that should minimize the risk of infection to laboratory staff especially in resource-limited settings.

\section{KEYWORDS}

Coronavirus; SARS-CoV-2; Medical Laboratory; Resource-limited setting, Good Laboratory Practice (GLP) 


\section{Introduction}

The outbreak of a novel coronavirus disease in Wuhan, China has spread to more than 200 countries worldwide. Severe acute respiratory syndrome coronavirus 2 (SARS-CoV-2) previously known by the provisional name 2019 novel coronavirus (2019-nCoV)[23], is a positive-sense single-stranded RNA virus. [12] It causes the respiratory illness known as coronavirus disease 2019 (COVID-19). SARS-CoV-2 is contagious in humans, and the World Health Organization (WHO) has designated the ongoing pandemic of COVID-19 a Public Health Emergency of International Concern [2].

Coronavirus can initiate an acute resolved disease but it can also be deadly, with a $2 \%$ case fatality rate. Severe disease onset might result in death due to massive alveolar damage and progressive respiratory failure. As of April 1st 2020, Globally 823,626 confirmed a case with 72,736 new cases within the last 24 hours has been reported. WHO has also reported 40,598 deaths and 4,193 within the last 24 hours [1]. Africa has the least cases with 4,073 confirmed cases and 287 within the last 24 hours and 91 deaths (with 14 death cases within the last 24 hours). Nigeria as at the time of this article has 111 confirmed cases with 20 new cases and 1 death case [27].

Considering the fragility of our health facilities in resource-limited settings and challenges with good laboratory practice for routine work coupled with poor implementation of health policies, it is pertinent that laboratory users should have some useful information that can be practicable in resource-limited settings like Africa to protect itself and prevent further spread of the virus. The overall statistics released by the WHO as regards the pandemic in Africa might not be a true reflection of what is obtainable on the ground as facts have revealed that access to testing in Africa is a big challenge. The current population of Nigeria is 204,842,081 as of Friday, April 3, 2020, based on Worldometer elaboration of the latest United Nations data but have less than 10 functional testing labs [10]. There might be more number of positives than is being reported. Clinical manifestation of patients presenting in the hospitals across the continent has been a consoling index as the number of deaths arising from SARS-CoV-2 complication in Africa has been very low as reported by WHO.

Given these, we have taken time to highlight necessary precautional measures to be taken based on good laboratory best practices across the various specialities of medical laboratory services that can be applicable in resource-limited settings to prevent the infection of laboratory staffs and further spread of the virus [3]. 
Safety in the medical/clinical laboratories is one of the very important pillars in laboratory procedures and medical practice. However, some determinants influence the safety of personnel, environment, and sample handling, some of which are the medical procedures carried out in the lab, use of PPE, the bio-safety level of the laboratory appropriate to the lab procedures and lots more. Considering the COVID 19 pandemic, several things must be done to ensure that the Medical Laboratory Scientist is protected, as well as the environment and community, not downplaying adherence to standard procedures, and ensuring quality management systems enforced.

Human-to-human transmissions of the novel coronavirus (2019-nCoV) have been described with incubation times between 2 and 10 days, facilitating its spread via droplets, contaminated hands or surfaces [17]. There may be additional forms of transmission that are yet not determined, hence laboratory scientist must treat samples as potentially harmful-highly infectious, if need be- till proven otherwise. Also, most patients in Africa resolve to self-medication when a fever is noticed before even presenting to the hospital due to readily accessible un-restricted access to over the counter pills in most African countries.

Generally, there is a wide range of disinfectants available that can be used to disinfect surfaces [19]. Kampf et al. [19] have noted that other coronaviruses, e.g. SARS and MERS, can persist on inanimate surfaces like metal, glass, or plastic for up to9days, but can be efficiently inactivated by surface disinfection procedures with $62-71 \%$ ethanol, $0.5 \%$ hydrogen peroxide, or $0.1 \%$ sodium hypochlorite within 1 min. Other biocidal agents such as $0.05 \%$ to $0.2 \%$ benzalkonium chloride or $0.02 \%$ chlorhexidine digluconate were less effective. Hence all workbenches across the various specialities must be appropriately disinfected before and after the close of work.

All Medical Laboratory Scientist should use 'standard precautions'. This includes proper hand hygiene, respiratory hygiene, and use of personal protective equipment (PPE), and injection safety practices when collecting samples [2].

\section{Diagnostic histopathology laboratory routine practices in the light of COVID-19 pandemic}

Histopathology laboratories are the designated laboratories where human tissues and cells are analysed for diagnostic, therapeutic, prognostic and forensic testing. The histopathology laboratory is fortunate in that routine Histotechnology processes often inactivate many viruses, e.g. Ebola [18]. 
The question then arises as to whether there is any evidence that these processes will have any effect on the activity of the coronavirus. Darnell et al. [10] determined that formalin and glutaraldehyde inactivated SARS-CoV in a temperature- and timedependent manner. While incubation at $4^{\circ} \mathrm{C}$ inhibited the effect of these chemicals, at $37^{\circ} \mathrm{C}$ or room temperature, formalin significantly decreased the infectivity of the virus on day 1 , while glutaraldehyde inactivated SARS-CoV after incubations of 1-2 days. Xu et al. [29] presented the autopsy findings of a 2019-nCoV patient. In this case study, core biopsies were taken from lung, liver and heart. Based on the quality of the accompanying photomicrographs, it seems that the core biopsies were fixed in formalin, processed through to paraffin and sections stained with H\&E. Duan et al. [13] found that several coronaviruses were made non-infectious after the following exposure times and temperatures: $90 \mathrm{~min}$ at $56^{\circ} \mathrm{C}, 60 \mathrm{~min}$ at $67^{\circ} \mathrm{C}$, and 30 $\min$ at $75^{\circ} \mathrm{C}$ [13]. Paraffin infiltration in most histopathology laboratories uses a temperature of $60-65^{\circ} \mathrm{C}$ for $2 \mathrm{~h}$ or more. It is; therefore, appropriate to consider that the formalin-fixed paraffin-embedded tissue block would have a low risk of coronavirus infectivity.

Based on the previous discussion, it appears prudent to refrain from performing frozen sections on possible cases of 2019-nCoV unless the laboratory is confident in containing aerosols in the cryostat. The same consideration should be applied to the grossing of partially fixed specimens. It appears, based on the limited autopsy study by Xu et al. [29], that only lung tissues exhibit microscopic evidence of 2019-nCoV infection whereas no viral change was noted in the liver and cardiac muscle. The few reports documenting the findings of COVID-19 show that the pathology is dominated by pulmonary findings, including pulmonary oedema and prominent proteinaceous exudates, vascular congestion, and intra-alveolar fibrinoid material and various degrees of an organization (fibroblastic plugs) corresponding to acute pulmonary injury patterns [2]. Also, there may be reactive type II pneumocyte hyperplasia, and atypical enlarged pneumocytes with large nuclei, amphophilic granular cytoplasm, and no definite intranuclear or cytoplasmic viral inclusions were identified [12]. The inflammatory infiltrate is predominantly lymphocytic without significant neutrophil participation. Immunohistochemistry for the Rp3 NP protein of SARS-CoV-2 showed staining of alveolar epithelial cells, including the damaged, desquamated cells present within alveolar spaces, but viral protein expression was only minimal in endothelial cells [2].

This finding is similar to that seen in MERS, where immunohistochemistry with four antibodies against MERS-CoV showed the presence of the virus scattered in cytokeratin-staining pneumocytes and syncytial cells, but not in CD68-positive macrophages [14] 


\section{Diagnostic Cytology laboratory routine practices in the light of COVID-19 pandemic}

Cytology samples most especially non-gynaecological and fine needle aspirate samples, on the other hand, are highly infective as these samples come fresh with no prior fixation for analysis. Hence in resource-limited settings such as Africa and Nigeria, at the level of hospitals and other health care institutions, it's therefore important firstly that decisions are made to prioritize the essential health care workers and reduce elective outpatient visits and inpatient admissions for elective interventions procedures or operations. It's a well-established fact that most of the cytology laboratory across Nigeria and most African countries alike are bio-safety 2 and below in structure and hence drastic alternative measures have to be taken. These measures are meant both to decrease the risk of infection to patients with routine annual and preventive health visits and elective procedures and to increase the available capacity of the hospitals in case of a large surge of infections [17]. Preventive measures to limit activities that can be safely postponed will undoubtedly affect the cytology laboratory, which will in-turn receive fewer specimens. This allows the cytology laboratory to re-evaluate their staffing needs and maybe change the workflow. Measures may include working in shifts, and staggered meal breaks, to avoid contact between people, and having only the strictly necessary in the laboratory.

The use of universal precautions [22] while it is important to know which cytology specimens can contain a viable and therefore transmissible virus, it is important to emphasize the use of universal standard precautions when dealing with any cytology specimen [22]. From the experience with SARS, we can extrapolate that SARS-CoV-2 can be present in faecal and urine samples, in addition to peripheral blood and respiratory samples. SARS-CoV-2 may be present in samples in patients without known COVID-19, in undiagnosed patients, in pre-symptomatic patients, in patients with asymptomatic or minimally symptomatic infections, and convalescent patients, who may still be shedding the virus [15]. From the available data, many, if not most, infections likely occur through contact with individuals who either don't have the disease or were not diagnosed with COVID-19.

Also, as with most specimens, the clinical diagnosis may not be indicated in the requisition. For all these reasons, all fresh specimens should be considered potentially infectious [22].

Special precautions should be taken in handling specimens and the preparation of which involves steps that can lead to aerosol formation. All technical procedures should be performed in a way that minimizes the generation of aerosols and droplets [17]. 
Preparatory steps that may generate aerosols or droplets include expelling aspirates from the needle or syringe, smearing the aspirated material, and potentially airdrying or heat drying the smears, in which pathologists, residents or cytoscientist may be involved during rapid on-site evaluation (ROSE). Air-drying or heat drying of smears is best performed under Class II Biosafety Cabinets (BSC) [25].

However in most cytology labs in resource-limited countries where this is not available good laboratory practise such as drying smears in enclosed coupling jars to reduce aerosols. Agitating the smears by hand or using handheld fans to speed up the drying of smears should be avoided. ROSE is an important measure to ensure the adequacy of specimens [25]. However, during an epidemic of a virus with respiratory-transmission, like SARS-CoV-2, clinical judgement should be used to determine if ROSE is necessary for the success of the biopsy procedure. If ROSE is performed, it should be performed with appropriate personal protective equipment (PPE) including gloves, laboratory coat/gown and goggles or face shields for eye protection and respiratory protection using a properly fit-tested filter respirator ( $\mathrm{N}$ 95 or higher level) or a powered air-purifying respirator (PAPR). The anticipated shortage of facemasks and filter respirators [52], makes very selective use of this procedure important, as we must reserve this equipment for essential patient encounters and procedures [26].

For similar reasons, it may be safer to suspend the activity of a pathologist-run FNA Clinic for the duration of the pandemic and consider performing FNAs only on a case-by-case basis, weighing the risks and benefits of the procedure for each patient. Cytopreparatory steps performed by scientist that can lead to aerosol/droplet formation include the opening of containers and removing tube caps, blending, vigorous shaking or mixing, vortexing, pipetting, aliquoting, diluting or centrifugation of fluids and discarding the supernatant.

All these cytopreparatory steps should be performed in Class II BSC, however, if this is not available air conditioning systems in the lab should be switched off and cross ventilation can help reduce this effect to some extent providing protection for the user, the sample, and the environment. The use of PPE, including gloves, gown, and face shield is also recommended for these procedures. Splash shields, sealed centrifuge rotors or sample cups are recommended for centrifugation; rotors and cups should be loaded and unloaded in a BSC51 [26].

The virus is inactivated by formalin and gamma irradiation. Therefore, according to the Centres of Disease Control and Prevention (CDC) guidelines [26], cytology laboratory activities, such as the pathologic examination and processing of formalinfixed or otherwise inactivated tissues (cell block preparations), and the routine staining and microscopic analysis of fixed smears are assigned biosafety level 2 (BSL2) [26]. 
This is the typical bio-safety level of all pathology laboratories and is assigned when working with agents associated with human diseases that pose a moderate health hazard or when working with any human-derived sample, including blood, body fluids, or tissue, in which the presence of an infectious agent is unknown. Most cytology specimens are fixed in either formalin or alcohol solutions with over $70 \%$ alcohol, which is considered effective to destroy this virus. However additional precautions, like the use of gloves may be indicated while handling and interpreting cytological preparations processed with these fixatives. Some pathologists may prefer using gloves for all their slides since glass slides are touched by multiple hands until they reach the pathologist's desk, and cannot be easily decontaminated. Although dipping the slides in $95 \%$ alcohol (or similar) for a couple of minutes would inactivate the virus, it would also erase the marks ("dots") on the slides. Other surface disinfectants, especially ones with short contact times, as mentioned below, can be tried to determine the practicality of their use on cytology slides.

General safe laboratory practices, especially procedures that are basic to good microbiological practices and procedures should be followed as recommended by the World Health Organization (WHO) [6] and the CDC [6] This includes training all personnel in the use of protective equipment, limiting access to the laboratory, frequent hand washing, and wearing personal protective equipment (PPE). Hand washing should be performed thoroughly with soap and water for at least 20 seconds. Alternatively, an alcohol-based hand sanitizer containing at least $62 \%$ alcohol can be used, if soap and water are not available. The use of PPE includes wearing gloves for all procedures, wearing a buttoned lab coat or gown, eye protection and, when needed, wearing a mask. Touching mucosal membranes of the eyes, nose and mouth should be avoided. Besides, because the virus can remain viable on surfaces (especially plastic and stainless steel surfaces) for up to 72 hours [25], it is recommended to decontaminate all work surfaces multiple times per day, using in-house prepared disinfectants or approved disinfectant solutions, wipes, towelettes or sprays.

In addition to benches, this includes decontamination of computer keyboards, phones, and frequently touched areas of microscopes [2]. The chemical substances involved include, among others, ethanol (minimum concentration of $62 \%-71 \%$ ), $0.5 \%$ hydrogen peroxide, quaternary ammonium, sodium hypochlorite $(0.1 \%)$, and a variety of acid solutions in various concentrations [19].

These substances should be used with the manufacturer-recommended disinfection directions and preparations for Human Coronavirus and the recommended contact time (i.e. the time the surface remains wet) which may range from 30 seconds to 10 minutes [19]. 


\section{Diagnostic haematology and blood transfusion science laboratory routine practices in the light of COVID-19 pandemic}

The haematology laboratory, on the other hand, is where blood and blood products are analysed [4]. Blood is one of the samples used to check for COVID 19 infection; it is important to strictly maintain universal precautions in sample handling. Considering a resource-limited setting like Nigeria, proper sample handling, transport and processing are important, despite weak health systems.

Facilities that offer to test for COVID 19 infection are just a handful in Nigeria, and getting tested is quite difficult, or almost impossible, considering present situations, hence it is the opinion of many scientists and professionals that the number of cases in Nigeria and most of Africa is under-reported, due to lack of testing facilities, denial of the existence of the virus, amongst other things.

A typical scenario which has played out; someone has had contact with an infected person in the past $7-12$ days, or has just returned from a country with a high rate of infection, he might have symptoms but decides to do home remedy - which is common in my clime--thinking it's a malaria infection, which is endemic in most parts of Africa. When conditions don't improve, he would inevitably go to the nearest health facility, which to a large extent is ill-prepared to attend to the patient. Health facilities, mostly government-owned institutions, due to limited/poorly managed resource-limited ill-prepared for such a pandemic plaguing the globe presently.

The medical laboratory is usually in the frontlines of this war; haematological analysis to check for red and white cell abnormalities and clotting anomalies are routine. With evidence of viral shedding, this poses a high risk to personnel and the environment [8]. Being a new pathogen, there are perhaps some still unknown characteristics, which has created some level of apprehension and uncertainty in routine analysis of suspected COVID 19 samples, and also a provision of blood free of SARS-CoV-2 pathogen [9].

Studies have shown that viral RNA can be detected in plasma and serum of patients infected with SARS-CoV at different times, after the onset of symptoms, but this isn't equivalent to the detection of intact infectious virus [8]. With more cases of asymptomatic infections among COVID 19 cases, there is the theoretical risk of transmission; hence ensuring blood safety is a worthy consideration.

\section{Diagnostic clinical chemistry laboratory routine practices in the light of COVID-19 pandemic}

Chemical pathology laboratory or clinical chemistry involves the study of biochemical processes in the body about the disease by analysis of body fluids such as blood and urine for diagnostic and therapeutic purposes [7]. 
Most chemical pathology laboratories in a resource-limited setting receive several samples such as whole blood, plasma, serum, urine, cerebrospinal fluid, pleural fluid and stool for analysis. However, the laboratory health care workers are exposed to a varying degree of infection ranging from sample collection, handling, processing, analysis and disposal of biological samples. The infrastructure of the laboratory should be by international best practices, GLP and adherence to policies and protocols of the Infection Prevention and Control Practices (IPAC) (25). The staff in the laboratory can become infected if appropriate precaution is not taken when handling biological samples' from patients with COVID-19. It is pertinent to note that in the wake of COVID-19 pandemic samples of different patients are sent routinely to health facilities which may pose a high risk to the laboratory health care workers. It becomes necessary that appropriate measures be taken by clinical laboratories to contain potentially infectious materials and forestall secondary infection and transmission (25).

As part of risk assessment, the physician is supposed to notify the laboratory staff when specimens from a patient with suspected or confirmed COVID-19 are submitted, through proper completion of request form or direct communication with laboratory. The case scenario however in a resource-limited setting is that most request forms sent are not properly filled as such difficult to know the true state of the patient and nature of the specimen. Also, the non-availability of appropriate containment levels, PPEs and waste disposal systems to mitigate the spread of infection is a major issue due to poor funding. This has exposed several laboratory workers to infection in the face of COVID-19 pandemic. Notwithstanding, with proper funding, provision of appropriate laboratory infrastructure, strict adherence to Good Laboratory Practice and WHO guidelines for transport and handling of infectious materials, there is a high possibility of reducing infection in a resource-limited setting (26).

As a general rule, all specimens received in the chemical pathology laboratory must be treated as a potentially high-risk specimen. To this end, laboratory workers must follow GLP from the point of reception, processing, analysis, storage and disposal.

This no doubt will help minimize the spread of infection to a great degree.

Some measures taken as GLP in a resource-limited setting is proper to use of PPEs such as laboratory coats, hand gloves, face masks, eye shield, shoe boots, appropriate bin liners for proper disposal of highly infectious, infectious and noninfectious materials and continuing education on Quality Management Systems There is ongoing research about the pathogenic potential and risk of transmission of the novel coronavirus and diseases it causes (4). It is therefore imperative for laboratory staff handling samples from suspected or confirmed cases of COVID-19 to follow the laboratory biosafety guidelines for the handling and transport of samples to prevent the spread of infection (26). 


\section{Diagnostic medical microbiology laboratory routine practices in the light of COVID- 19 pandemic}

The diagnostic microbiology laboratory is primarily involved in the diagnosis of infectious disease. It plays key roles in isolation/detection of the etiologic agent, evidence-based recommendation on antimicrobial options, monitoring the effectiveness of therapeutic regimes, surveillance of infectious agents and investigation of disease outbreaks (5). Expectedly, various types of specimens are received, the choice of which is determined by the requesting physician and the clinical site of infection. In the light of the COVID-19 pandemic, some specimens must be deemed high risk by requesting physician, laboratory scientists, technologists and technicians. Such specimens must be collected, transported, handled with the requisite caution without prejudice to good laboratory practice (GLP) to minimize the risk of infection to the aforementioned professionals and other laboratory staff $(6,22)$.

Respiratory specimens which include oropharyngeal swabs nasopharyngeal swabs, nasopharyngeal wash, sputum, tracheal aspirate, broncheoalveolar lavage (BAL) fluid, pleural fluid or aspirate must be accorded high risk especially when the clinical suspicion of COVID-19 accompanies the request. Although SARS-CoV-2 has not been detected in urine or other specimens, the zoonotic strain, Middle East respiratory syndrome coronavirus (MERS-CoV) has been detected in a variety of human specimens including urine, faeces, blood and other body fluids (6). In the absence of contrary scientific evidence, it is safe for laboratory personnel to take appropriate precautions when handling these specimens from patients with SARS-CoV-2 or suspects to minimize the risk of spread of infection accidentally. Laboratory procedures which generate aerosols such as centrifugation, grinding, blending, and vigorous shaking, mixing, sonic disruption and opening of containers of infectious materials whose internal pressure may be different from the ambient pressure should be carried out in a safety cabinet (4).

The challenge of COVID-19 pandemic concerning diagnostic microbiology laboratories may be more pronounced in resource-limited countries even though most facilities in are containment levels 1 and 2 (3). Such settings are characterized by the government's poor funding of healthcare, substandard equipment/reagents and absence of a proper regulatory framework for laboratory practices. These challenges may lead to a shortage of PPEs, reagents, equipment, vacuous protocols/procedures which may not take the safety of laboratory personnel into consideration and unreliable laboratory results.

As the figures of infected persons in most African countries show a gradual rise when compared with her first-world counterparts in Europe and North America where figures are alarmingly high, these peculiar challenges must be taken into account and laboratory personnel must continuously strive to build capacity. 
The need for coordination and synergy of laboratories at local, state and federal levels cannot, therefore, be overemphasized.

\section{Invitro-fertilization (IVF) laboratory routine practices in the light of COVID-19 pandemic}

Successful and consistent outcomes in human in-vitro fertilization (IVF) is vital and can be readily achieved by all IVF clinics through consideration and optimization of each procedure associated with the collection and processing of gametes, culminating in the resultant culture and transfer of healthy embryos [27]. Laboratory safety is an important and critical component to the success of in-vitro fertilization. The IVF laboratory serves the general public and Nigerians in Diaspora who prefers to carry out IVF procedure back home due to success rates and affordable cost. As earlier reported the index case and many other individuals who tested positive to CONVID-19 in Nigeria were mainly individuals who came in from highly endemic regions. It may hence be important that some major activities be suspended to curtail the spread of the virus [11]. However, for prioritised cases, professional safety measures and practices for Patients who are prepared for an invitro fertilization procedure which will involve series of laboratory tests, scans and assessments before the procedure must be adhered to. Due to the peculiarity of this unit which requires a multidisciplinary approach comprising a team of health care professionals which include doctors, nurses, embryologist, laboratory scientist and other staffs of the unit, all professionals must adhere to stipulated regulations and guidelines by WHO and CDC which can be accessed from (https://www.cdc.gov/coronavirus/2019-nCoV/lab/lab-biosafety-guidelines.html) and (Africa CDC) African Union Commission health care workers guidelines for coronavirus.

As an embryologist/medical laboratory scientist, sophisticated biomedical instrument and technology, computers and methods requiring manual dexterity to perform laboratory testing on blood fluids. These include blood samples for hormonal assay, and general screening test, semen for seminal fluid analysis. Proper care and good laboratory practise to prevent exposure to coronavirus (COVID-19) should be ensured [27].

It's therefore important that Laboratory staffs should ensure the proper use of personal protective equipment's (PPE), follow protocols for appropriate chemical hygiene plan (CHP) and use of biological safety cabinets in the in-vitro fertilization laboratory. 


\section{REFERENCES}

1. "About Novel Coronavirus (2019-nCoV)". United States Centers for Disease Control and Prevention (CDC). 11 February 2020. Archived from the original on 11 February 2020. Retrieved 25 February 2020.

2. Alsaad KO, Hajeer AH, Al Balwi M, et al. Histopathology of Middle East respiratory syndrome coronovirus (MERS-CoV) infection - clinicopathological and ultrastructural study. Histopathology. 2018; 72: 516-524.

3. Ahmed SS, Alp E, Ulu-Kilic A, Doganay M. Establishing molecular microbiology facilities in developing countries. Journal of Infection and Public Health 2015; 8(6): 513-525. https://doi.org/10.1016/i.jiph.2015.04.029

4. Baral, R (2014). Pathology Laboratories and Infection Prevention and Control. Journal of Pathology of Nepal, 4(8):1

5. Benbachir, M. (2008). "Role of the microbiology laboratory in infection control," in Guide to Infection Control in Hospitals, IV Edn, eds R. Wenzel, G. Bearman, T. Brewer, and J.-P. Butzler (Boston: International Society for Infectious Diseases).

6. Biosafety in Microbiological and Biomedical Laboratories, 5th edition, Centers for Disease Control and Prevention and National Institutes of Health, February 2007. http://www.cdc.gov/biosafety/

7. Burtis, C.A., Ashwood, E.R., and Bruns, D.E (2006). Tietz textbook of clinical chemistry (4th ed.). Saunders, Pp 2448

8. Centre for Disease Control. Coronavirus Disease 2019 (COVID-19): Laboratory Biosafety Guidelines. Reviewed 20th March 2020

9. "CoV2020". GISAID EpifluDB. Archived from the original on 12 January 2020. Retrieved 12 January 2020.

10. Emorinken, Moses (3 April 2020). "COVID-19: Nigeria gets additional labs, tests 4,000". The Nation Newspaper. Retrieved 3 April 2020. 
11.https://africacdc.org/download/2019-novel-coronavirus-disease-outbreakwhat-health-care-workers-should-know/ Retrieved 1 April 2020.

12.Darnell ME, Subbarao K, Feinstone SM, et al. Inactivation of the coronavirus that induces severe acute respiratory syndrome, SARS-CoV. J Virol Methods. 2004 Oct 1;121(1):85-91.

13.Duan SM, Zhao XS, Wen RF, et al. Stability of SARS coronavirus in human specimens and environment and its sensitivity to heating and UV irradiation. Biomed Environ Sci. 2003 Sep;16(3):246-255.

14.Gorbalenya AE. Severe acute respiratory syndrome-related coronavirus-The species and its viruses, a statement of the Coronavirus Study Group. bioRxiv. 2020 Jan 1.

15.Guarner J. Three emerging Coronaviruses in two decades the story of SARS, MERS, and now COVID-19. Editorial Am J Clin Pathol. 2020 Feb 13. DOI:10.1093/AJCP/AQAA029

16. "Healthcare Professionals: Frequently Asked Questions and Answers". United States Centers for Disease Control and Prevention (CDC). 11 February 2020. Archived from the original on 14 February 2020. Retrieved 15 February 2020

17. Henwood A. A survival guide for laboratory professionals. Scotts Valley, CA, USA: Amazon Create Space Independent Publishing Platform; 2019. Chapter 18 Disinfection, 149-155.

18. Henwood AF. Ebola and histotechnologists. J Histotechnology. 2018 Apr 3;41(2):71-73.

19. Kampf G, Todt D, Pfaender S, et al. Persistence of coronaviruses on inanimate surfaces and its inactivation with biocidal agents. J Hosp Infect. 2020. DOI:10.1016/j.jhin.2020.01.022

20.Paules $\mathrm{Cl}$, Marston HD, Fauci AS. Coronavirus infections-more than just the common cold. JAMA. 2020 Jan 23. doi: 10.1001/jama.2020.0757

21.Perlman S. Another decade, another coronavirus. N Engl J Med. 2020 Jan 24;382:760-762. 
22.Public Health England. 2020. COVID-19: safe handling and processing for samples in laboratories.

Available at https://www.gov.uk/government/publications/wuhan-novel-coronavirusguidance-for-clinical-diagnostic-laboratories/wuhan-novel-coronavirushandling-and-processing-of-laboratory-specimens.

23. World Health Organization 2004. Laboratory biosafety manual. - 3rd ed.

24."WHO Director-General's opening remarks at the media briefing on COVID-19 - 11 March 2020". World Health Organization (WHO) (Press release). 11 March 2020. Archived from the original on 11 March 2020. Retrieved 12 March 2020.

25.World Health Organization. Guidance on COVID-19: Safe handling and processing for samples in laboratories. Public Health England. Updated 28th March 2020

26. World Health Organization. Guidance on regulations for the transport of infectious substances 2019-2020. (online) 2019. (cited: January 28, 2020) https://www.who.int/ihr/publications/WHO-WHE-CPI-2019.20

27.Sakkas D, Balaban B, Gardner DK. 2014: Laboratory procedures for human in vitro fertilization. Semin Reprod Med. 32(4):272-82. doi: 10.1055/s-00341375179.

28. World Health Organization; 2020, pp 1-5. https://www.who.int/publicationsdetail/infection-prevention-and-control-during-health-care-when-novelcoronavirus-(ncov)-infection-is-suspected-20200125

29.Xu Z, Shi L, Wang Y, et al. Pathological findings of COVID-19 associated with acute respiratory distress syndrome. Lancet Respir Med. 2020. published online Feb 17. DOI:10.1016/S2213-2600(20)30076-X 
30.Zhu N, Zhang D, Wang W, et al. A novel coronavirus from patients with pneumonia in China, 2019. N Engl J Med. Feb 20, 2019. 382:727-733. DOI:10.1056/NEJMoa2001017

31.Zhou F, Yu T, Du R, et al. Clinical course and risk factors for mortality of adult inpatients with COVID-19 in Wuhan, China: a retrospective cohort study. Lancet. 2020. 